\title{
SATURATED BOUNDARY FEEDBACK STABILIZATION OF A LINEAR WAVE EQUATION*
}

\author{
CHENG-ZHONG XU ${ }^{\dagger}$ AND GEN QI XU ${ }^{\ddagger}$
}

\begin{abstract}
In this paper, we study boundary feedback stabilization of a linear wave equation by saturated linear or nonlinear Neumann control laws. Firstly we prove asymptotic stabilization of the closed-loop system when the feedback control law has a linear growth rate around zero. In particular, we study the effect of spatial dimension on the decay rate of the closed loop system. More precisely, we prove that in the one-dimensional (1D) case the smooth solutions of the closed-loop system decay exponentially to zero as $t \rightarrow \infty$; in the two-dimensional case the smooth solutions decay asymptotically to zero faster than any polynomial $(1 / t)^{\alpha} \forall \alpha>0$; and in the three-dimensional case the smooth solutions decay to zero like $(1 / t)^{2}$ as $t \rightarrow \infty$. Secondly we study robustness of the stabilization faced with the boundary disturbances. We show that, in the 1D case, every solution of the closed-loop system decays asymptotically to zero provided that the unknown disturbance is in the Sobolev space $W^{1,1}(0, \infty)$. Finally we consider a sliding mode output feedback control law that is regarded as the limit case of the Yosida approximation of the sign function. We prove that the resulting system is not asymptotically stable. However each smooth solution of the resulting system is bounded and converges asymptotically to a periodic solution as $t \rightarrow \infty$.
\end{abstract}

Key words. wave equation, saturated nonlinear feedback stabilization, boundary disturbance, sliding mode control, periodic trajectories, asymptotic stability

AMS subject classifications. 93C10, 93C20, 93D15, 93D30

DOI. $10.1137 / 15 \mathrm{M} 1034350$

1. Introduction. In this paper, we consider a control problem of one-dimensional (1D) linear wave equation with unknown boundary disturbance as follows:

$$
\left\{\begin{array}{l}
w_{t t}(x, t)=w_{x x}(x, t), \quad x \in(0,1), \quad t>0 \\
w(0, t)=0, \quad w_{x}(1, t)=u(t)+d(t), \quad t>0, \\
w(x, 0)=w_{0}(x), \quad w_{t}(x, 0)=w_{1}(x), \quad x \in(0,1)
\end{array}\right.
$$

where $u(t)$ is the control and $d(t)$ is the disturbance assumed bounded and continuous on $[0, \infty)$. Suppose that the collocated information is valid, i.e., $y(t)=w_{t}(1, t)$. Then the closed-loop system by the output feedback $F$ is described by

$$
\left\{\begin{array}{l}
w_{t t}(x, t)=w_{x x}(x, t), x \in(0,1), \quad t>0 \\
w(0, t)=0, \quad w_{x}(1, t)=-F\left(w_{t}(1, t)\right)+d(t), \\
w(x, 0)=w_{0}(x), \quad w_{t}(x, 0)=w_{1}(x), \quad x \in(0,1),
\end{array}\right.
$$

where $F: \mathbb{R} \rightarrow \mathbb{R}$ is a continuous monotone increasing function satisfying $F(s) s>0$ $\forall s \neq 0$ and $F(0)=0$.

$F$ is said to be a saturated linear function if there are some positive constants $M_{1}, M_{2}, C_{1}$, and $C_{2}$ such that the following conditions are satisfied:

$$
\begin{cases}C_{1}|\sigma| \leq|F(\sigma)| \leq C_{2}|\sigma| & \forall|\sigma|<M_{1} \\ |F(\sigma)| \leq M_{2} & \forall|\sigma| \geq M_{1}\end{cases}
$$

* Received by the editors August 7, 2015; accepted for publication (in revised form) November 26, 2018; published electronically January 22, 2019.

http://www.siam.org/journals/sicon/57-1/M103435.html

Funding: This research was supported by the National Natural Science Foundation in China (NSFC-61773277).

${ }^{\dagger}$ University of Lyon, LAGEP, Bâtiment CPE, Université Lyon 1, F-69622, Villeurbanne Cedex, France (xu@lagep.univ-lyon1.fr).

${ }_{\ddagger}^{\ddagger}$ Department of Mathematics, Tianjin University, Tianjin 300350, China (gqxu@tju.edu.cn). 
That means $F$ has linear growth rate around zero and bounded by some constant $M_{2}$ at infinity. A simple example of the saturated linear function is

$$
F(\sigma)=\left\{\begin{array}{cl}
M \operatorname{sign}(\sigma) & \text { if }|\sigma| \geq M \\
\sigma & \text { if }|\sigma|<M
\end{array}\right.
$$

where $M$ is a positive real number and $\operatorname{sign}(\sigma)=\frac{\sigma}{|\sigma|}, \sigma \neq 0$.

Usually the function $\operatorname{sign}(x)$ is defined as $\operatorname{sign}(x)=\frac{x}{|x|}$ if $x \neq 0$ and $\operatorname{sign}(0)=$ $[-1,1]$. The Yosida approximation of $M \operatorname{sign}(x)$ is $F_{\lambda}(x)$ defined by, for $\lambda>0$,

$$
F_{\lambda}(x)=\left\{\begin{aligned}
M \operatorname{sign}(x) & \text { if }|x|>\lambda M \\
\lambda^{-1} x & \text { if }|x| \leq \lambda M
\end{aligned}\right.
$$

Clearly, $F_{\lambda}(x)$ is a saturated linear function for $\lambda>0$. Note that $\lim _{\lambda \rightarrow 0} F_{\lambda}(x)=$ $M \operatorname{sign}^{0}(x)$, where $\operatorname{sign}^{0}(x)$ denotes the element $z \in \operatorname{sign}(x)$ of the least norm. Therefore, as a limit case of saturated control laws, we further consider the sliding mode feedback controlled system (1.1), and the associated closed-loop system described by

$$
\left\{\begin{array}{l}
w_{t t}(x, t)=w_{x x}(x, t), \quad x \in(0,1), \quad t>0, \\
w(0, t)=0, \quad w_{x}(1, t) \in-M \operatorname{sign}\left(w_{t}(1, t)\right)+d(t), \\
w(x, 0)=w_{0}(x), \quad w_{t}(x, 0)=w_{1}(x), \quad x \in(0,1) .
\end{array}\right.
$$

In the present paper we are specifically interested in the feedback stabilization of (1.1) by the saturated control law in (1.3). Secondly we are interested in whether or not the stability of the system with the sliding mode control law can be deduced from that of the system with the saturated feedback control.

Mathematically speaking the systems (1.2) and (1.6) are nonlinear and nonautonomous because of the presence of the disturbance $d(t)$. In the literature, the wave equation with nonlinear boundary feedback control has been extensively studied (cf. [11], [17], [5], [6], [26], and [27] to cite only a few). It has been proved in [26] and [27] that, to obtain uniform exponential stabilization of the wave equation, the nonlinear boundary velocity feedback must have a linear growth not only around zero but also at infinity. Vancostenoble and Martinez [26] have proved that, with the nonlinear feedback (1.4), the energy decay rate being nonuniform depends on the initial condition. This fact has been illustrated by a recent work of Prieur, Tarbouriech, and Gomes Da Silva [20].

Unlike the standard feedback control problem where the goal is to find a linear stabilizing feedback control law, in the present paper we restrict ourselves to the case where the control has the a priori constraint $\|u\|_{L^{\infty}} \leq M, M>0$. The constraint necessitates a choice of nonlinear feedback law which drives the state $\left(w, w_{t}\right)$ to zero as $t \rightarrow \infty$. In a neighborhood of zero the feedback law may be nonlinear but is bounded below and above by some linear functions (see the condition (1.3)). Inspired by the work of Slemrod [24] (see also Chen [4], Conrad and Pierre [7], and Rao [21]), we propose saturated feedback control laws to asymptotically stabilize the system around the origin. Thanks to the result in [27] and [26], with the saturated feedback control law, the nonuniform exponential decay is the best decay rate to be expected. In the present paper, our main results are stated as follows.

(1) In absence of the disturbance, the smooth solutions of the system (1.2) with the saturated feedback control (1.3) decay exponentially to zero in the energy space, as time goes to infinity. Specifically, the decay rate of smooth solutions to the wave equation changes with the spatial dimension. 
Due to the result in [27] and [26], we have no uniform exponential stability of the closed-loop system (1.2) since $F$ has no linear growth at infinity. However, for smooth initial conditions we still get exponential decay of the solutions to zero, and the exponential decay rate depends on the initial condition. Vancostenoble and Martinez [26] have proved that, with the nonlinear feedback (1.4), the decay rate being nonuniform depends on the initial condition. Our result is established for the general nonlinear feedback (1.3).

Different from the 1D case, we prove that, in the two-dimensional (2D) case, the smooth solutions decay asymptotically to zero faster than any polynomial $(1 / t)^{\alpha}$ $\forall \alpha>0$ and that the smooth solutions decay to zero like $(1 / t)^{2}$ as $t \rightarrow \infty$ in the three-dimensional (3D) case.

(2) In presence of the disturbance, for the nonlinear feedback (1.3), the solutions to the system (1.2) decay asymptotically to zero in the energy space, provided that $d \in W^{1,1}(0, \infty)$.

(3) As a limit case of the Yosida approximation of sign function we consider the sliding mode output feedback control law. We prove that, even in absence of the disturbance, the closed-loop system (1.6) is nonasymptotically stable in the energy space. However every smooth solution of the resulting system is bounded and converges asymptotically to a periodic solution as $t \rightarrow \infty$. Similarly, in presence of the disturbance, each smooth trajectory of the closed-loop system is bounded and converges asymptotically to a periodic trajectory whose energy is constant and smaller or equal to $M^{2}$.

(4) Finally the saturated feedback stabilized $1 \mathrm{D}$ wave equation is shown to be robust faced with the boundary disturbance from the Sobolev space $W^{1,1}(0, \infty)$.

From point (3) we see that the solution of the closed-loop system by the saturated feedback control does not converge to that of the sliding mode feedback controlled system as $\lambda \rightarrow 0$. To the best of our knowledge, the results on the saturated feedback control are new for the high-dimensional wave equation.

The rest of the paper is organized as follows. In section 2 we discuss the boundary stabilization of the wave equation by the saturated feedback control law in (1.3). We prove that the closed-loop system is globally asymptotically stable in the cases 1D, $2 \mathrm{D}$, and 3D. We estimate the decay rate of the smooth solutions for the closed-loop system. In section 3 , by using the LaSalle principle, we prove asymptotic stability of the closed-loop system in the energy space and that holds independent of unknown disturbances in the Sobolev space $W^{1,1}\left(\mathbb{R}_{+}\right)$. In section 4 , the sliding mode control law is taken as the limit of a sequence of saturated feedback control laws. Existence and uniqueness of the solutions are established in the energy space by using the maximal monotone operator technique. The long-time asymptotic behavior of the closed-loop system is described in detail. In particular we prove that each regular trajectory is bounded and converges asymptotically to a periodic one. Section 5 is devoted to our conclusions.

2. Boundary feedback stabilization with saturated control laws. In this section we consider the boundary feedback stabilization of a wave equation with saturated control laws in (1.3).

2.1. 1D wave equation. In this subsection we study the stability of the closedloop system governed by the following $1 \mathrm{D}$ wave equation:

$$
\left\{\begin{array}{l}
w_{t t}(x, t)=w_{x x}(x, t), \quad x \in(0,1), \quad t>0 \\
w(0, t)=0, \quad w_{x}(1, t)=-F\left(w_{t}(1, t)\right), \quad t>0, \\
w(x, 0)=w_{0}(x), \quad w_{t}(x, 0)=w_{1}(x), \quad x \in(0,1),
\end{array}\right.
$$


where the nonlinear feedback $F: \mathbb{R} \rightarrow \mathbb{R}$ is continuous, increasing, and satisfying the condition (1.3). To study asymptotic stability of the system (2.1) we need to accomplish two tasks: (i) prove existence and uniqueness of its solutions in some Hilbert space; (ii) guarantee required regularity for smooth trajectories.

We take the state space to be the real Hilbert space $X=H_{L}^{1}(0,1) \times L^{2}(0,1)$ suggested by the energy of the system, where $H_{L}^{1}(0,1)=\left\{g \in L^{2}(0,1) \mid g^{\prime} \in\right.$ $\left.L^{2}(0,1), g(0)=0\right\}$. By $\langle f, g\rangle_{X}$ we denote the inner product of $f, g \in X$. The induced Hilbert norm is given by the energy

$$
\|f\|_{X}^{2}=\int_{0}^{1}\left[f_{1 x}^{2}(x)+f_{2}^{2}(x)\right] d x .
$$

The nonlinear contraction semigroup theory (cf. [3], [10], [2], [13]) is made use of to fulfil (i) and (ii).

Define the nonlinear operator $\tilde{A}: \mathcal{D}(\tilde{A}) \rightarrow X$ such that

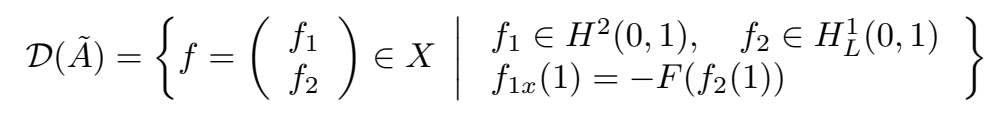

and

$$
\tilde{A} f=\left(\begin{array}{c}
-f_{2} \\
-f_{1 x x}
\end{array}\right) \forall f \in \mathcal{D}(\tilde{A}) .
$$

We set $\phi_{1}(t)=w(x, t)$ and $\phi_{2}(t)=w_{t}(x, t)$, and $\phi(t)=\left(\phi_{1}(t), \phi_{2}(t)\right)^{T}$. Then the state equation of the system (2.1) is written as follows:

$$
\dot{\phi}+\tilde{A} \phi=0, \quad \phi(0)=\phi^{0} \in X .
$$

Lemma 1. The operator $\tilde{A}$ defined by $(2.2)-(2.3)$ is maximal monotone, $\mathcal{D}(\tilde{A})$ is a dense set in $X$, and the resolvent operator $(I+\lambda \tilde{A})^{-1}$ is compact $\forall \lambda>0$.

Proof. The operator $\tilde{A}$ is monotone if and only if $\langle\tilde{A} f-\tilde{A} g, f-g\rangle_{X} \geq 0 \forall f, g \in$ $\mathcal{D}(\tilde{A})$. Indeed, by integration by parts we have

$$
\begin{aligned}
\langle\tilde{A} f-\tilde{A} g, f-g\rangle_{X} & =\left\langle\left(\begin{array}{c}
-f_{2}+g_{2} \\
-f_{1 x x}+g_{1 x x}
\end{array}\right),\right. \\
\left.\left(\begin{array}{c}
f_{1}-g_{1} \\
f 2-g_{2}
\end{array}\right)\right\rangle_{X} & =\left.\left(g_{2}-f_{2}\right)\left(f_{1 x}-g_{1 x}\right)\right|_{0} ^{1} .
\end{aligned}
$$

Using the boundary condition we get easily

$$
\left.\langle\tilde{A} f-\tilde{A} g, f-g\rangle_{X}=\left(g_{2}(1)\right)-f_{2}(1)\right)\left[F\left(g_{2}(1)\right)-F\left(f_{2}(1)\right)\right] .
$$

As the function $F$ is monotone increasing on $\mathbb{R}$, it follows from (2.5) that the inner product is positive or zero, and hence $\tilde{A}$ is monotone.

Since $\mathcal{D}(\tilde{A})$ contains $C_{0}^{\infty}(0,1)$ (the set of infinitely differential functions with compact support in $(0,1))$, so it is dense in $X$.

To show that $\tilde{A}$ is maximal, it is sufficient to prove that $\operatorname{Ran}(I+\tilde{A})=X$. Let $g \in X$. We solve the equation $(I+\tilde{A}) f=g$ for some $f \in \mathcal{D}(\tilde{A})$. Equivalently we solve $f_{2}=f_{1}-g_{1}$ and

$$
\begin{aligned}
& f_{1}-f_{1 x x}=g_{1}+g_{2}, \\
& f_{1}(0)=0, \\
& f_{1 x}(1)=-F\left(f_{2}(1)\right) .
\end{aligned}
$$

Copyright (c) by SIAM. Unauthorized reproduction of this article is prohibited. 
We set $\tilde{g}_{12}(x)=g_{1}(x)+g_{2}(x)$. The second order differential equation (2.6) has a unique solution, given by

$$
\begin{gathered}
f_{1}(x)=\sinh (x) \eta^{0}+\int_{0}^{x} \sinh (x-\xi) \tilde{g}_{12}(\xi) d \xi \\
f_{2}(x)=f_{1}(x)-g_{1}(x)
\end{gathered}
$$

where $\eta^{0}$ is the unique constant determined by the equation

$$
\begin{aligned}
& \cosh (1) \eta^{0}+\int_{0}^{1} \cosh (1-\xi) \tilde{g}_{12}(\xi) d \xi \\
& \quad=-F\left(\sinh (1) \eta^{0}+\int_{0}^{1} \sinh (1-\xi) \tilde{g}_{12}(\xi) d \xi-g_{1}(1)\right) .
\end{aligned}
$$

We check that $f_{1} \in H^{2}(0,1) \cap H_{L}^{1}(0,1)$ and $f_{2}=f_{1}-g_{1} \in H_{L}^{1}(0,1)$. Hence $\operatorname{Ran}(I+$ $\tilde{A})=X$, i.e., $\tilde{A}$ is maximal.

From the above we see that $(I+\tilde{A})^{-1}$ maps a bounded set in $X$ into a bounded set in $H^{2}(0,1) \times H^{1}(0,1)$. Since the injections $H^{2}(0,1) \hookrightarrow H^{1}(0,1)$ and $H^{1}(0,1) \hookrightarrow$ $L^{2}(0,1)$ are compact, the resolvent operator $(I+\tilde{A})^{-1}$ is compact. Hence every resolvent operator $(I+\lambda \tilde{A})^{-1}$ is compact for $\lambda>0$.

THEOREM 1. (i) The operator $(-\tilde{A})$ generates a semigroup of contractions $(\mathbb{T}(t))_{t \geq 0}$ on $X$. (ii) For each $\phi^{0} \in X$ the state equation (2.4) has a unique solution $\phi(t)$ in $C([0, \infty), X)$, given by $\phi(t)=\mathbb{T}(t) \phi^{0}$. (iii) If $\phi^{0} \in \mathcal{D}(\tilde{A})$, the unique solution $\phi(t) \in \mathcal{D}(\tilde{A})$ is differentiable at a.e. $t>0$, and $(2.4)$ is satisfied at a.e. $t>0$ in $L^{\infty}((0, \infty), X)$. (iv) The orbit (or trajectory) $\gamma\left(\phi^{0}\right)$ through $\phi^{0} \in X$ such that $\gamma\left(\phi^{0}\right)=\left\{\mathbb{T}(t) \phi^{0} \mid t \geq 0\right\}$ is precompact. (v) The zero solution of (2.4) is globally asymptotically stable in $X$ such that $\lim _{t \rightarrow \infty} \mathbb{T}(t) \phi^{0}=0 \forall \phi^{0} \in X$.

Proof. The statements (i)-(iv) are directly proved by applying Lemma 1 and the theory of nonlinear semigroups (cf. [10], [3], [2], [13], [5]).

(v) The zero solution of (2.4) is Lyapunov stable since the mapping $\mathbb{T}(t)$ is a contraction. To prove asymptotic stability it is sufficient to prove that $\mathbb{T}(t) \phi^{0}$ tends to zero as $t \rightarrow \infty \forall \phi^{0} \in \mathcal{D}(\tilde{A})$. Take an initial condition $\phi^{0} \in \mathcal{D}(\tilde{A})$ and a Lyapunov function candidate $V(\phi)=\|\phi\|_{X}^{2}$. By (iii) and differentiating $V(\phi(t))$ along the trajectory $\phi(t)=\mathbb{T}(t) \phi^{0}$ we get

$$
\dot{V}(\phi(t))=-2\langle\tilde{A} \phi(t), \phi(t)\rangle_{X}=-2 \phi_{2}(1, t) F\left(\phi_{2}(1, t)\right) \leq 0 \text { a.e. } t>0
$$

or

$$
V(\phi(t))=V\left(\phi^{0}\right)-\int_{0}^{t} 2 \phi_{2}(1, \tau) F\left(\phi_{2}(1, \tau)\right) d \tau .
$$

Hence the energy is decreasing with time, and so we have $\lim _{t \rightarrow \infty} V(\phi(t))=c$.

Since the trajectory is precompact, the $\omega$-limit set $\omega\left(\phi^{0}\right)$ is not empty and contained in $\mathcal{D}(\tilde{A})$. Moreover the $\omega$-limit set is contained in a constant energy set $E_{c}$ for some $c \geq 0$,

$$
E_{c}=\left\{f \in \mathcal{D}(\tilde{A}) \mid V(f)=c, f_{2}(1)=0\right\} .
$$

Knowing that the $\omega$-limit set is invariant under the semigroup $(\mathbb{T}(t))_{t \geq 0}$, we apply the multiplier method to prove that it is reduced to a single point, i.e., $\omega\left(\phi^{0}\right)=\{0\}$. 
Indeed, take anyone $\varphi \in \omega\left(\phi^{0}\right)$. As $\varphi \in \mathcal{D}(\tilde{A})$, the trajectory $\mathbb{T}(t) \varphi$ is differentiable at a.e. $t>0$ and $\mathbb{T}(t) \varphi \in \mathcal{D}(\tilde{A}) \forall t>0$. Let $\left(w(\cdot, t), w_{t}(\cdot, t)\right)^{T}=\mathbb{T}(t) \varphi$. Then the trajectory $\left(w(\cdot, t), w_{t}(\cdot, t)\right)$ satisfies the following PDE:

$$
\left\{\begin{array}{l}
w_{t t}(x, t)=w_{x x}(x, t), \quad x \in(0,1), \quad t>0, \\
w(0, t)=0, \quad w_{x}(1, t)=0, \quad w_{t}(1, t)=0, \quad t>0, \\
w(x, 0)=\varphi_{1}(x), \quad w_{t}(x, 0)=\varphi_{2}(x), \quad x \in[0,1] .
\end{array}\right.
$$

Using the multiplier $x w_{x}$ in $(2.7)$ we get

$$
\int_{0}^{T} w_{t}^{2}(1, t) d t=\int_{0}^{T} \int_{0}^{1}\left(w_{x}^{2}+w_{t}^{2}\right) d x d t+\left.2 \int_{0}^{1}\left(w_{t} x w_{x}\right)\right|_{0} ^{T} d x \forall T>0 .
$$

As the energy is constant along the trajectory, by using the Cauchy inequality in (2.8) we get the following:

$$
(T-2) V(\varphi) \leq \int_{0}^{T} w_{t}^{2}(1, t) d t \leq(T+2) V(\varphi) \forall T>0 .
$$

By taking $T>2$ and the fact that $w_{t}(1, t)=0$, it is deduced that $\varphi=0$. Hence $\omega\left(\phi^{0}\right)=\{0\}$. Therefore every trajectory $\mathbb{T}(t) \phi^{0}$ converges to zero as $t \rightarrow \infty \forall \phi^{0} \in X$ (cf. Theorem 1.1.8, p. 12 of [14]). The proof of Theorem 1 is complete.

As shown in [27] and [26], to obtain uniform exponential stabilization of the wave equation the nonlinear boundary velocity feedback must have a linear growth not only around zero but also at infinity. Since we do not have linear growth at infinity, we have no uniform exponential stability of the closed-loop system (2.4). If the initial data has certain smoothness, we want to know whether or not the solution decays exponentially. The following theorem gives an answer.

Theorem 2. For each initial condition $\phi^{0} \in \mathcal{D}(\tilde{A})$ the solution of the system (2.4) decays exponentially to zero in $X$ as $t \rightarrow \infty$.

Proof. Consider the Lyapunov function candidate $E: X \rightarrow \mathbb{R}^{+}$(cf.[8], [12], [18]) such that

$$
E_{\epsilon}(w, v)=E(w, v)+\epsilon \rho(w, v)
$$

where

$$
E(w, v)=\frac{1}{2} \int_{0}^{1}\left(w_{x}^{2}+v^{2}\right) d x, \quad \rho(w, v)=2 \int_{0}^{1} x v w_{x} d x
$$

and

$$
\epsilon=\min \left(\frac{1}{4}, \frac{C_{1}}{1+C_{2}^{2}}\right),
$$

where the constants $C_{1}$ and $C_{2}$ are defined as in (1.3). It is easy to see that

$$
\frac{1}{2} E(w, v) \leq E_{\epsilon}(w, v) \leq \frac{3}{2} E(w, v) .
$$

Since $\phi^{0} \in \mathcal{D}(\tilde{A})$, the solution $\left(w(\cdot, t), w_{t}(\cdot, t)\right)^{T}=\mathbb{T}(t) \phi^{0}$ is a strong solution to (2.4) or a smooth solution to the PDE (2.1). For the sake of simplicity we set $E_{\epsilon}(t)=E_{\epsilon}\left(w(\cdot, t), w_{t}(\cdot, t)\right)$ and $E(t)=E\left(w(\cdot, t), w_{t}(\cdot, t)\right)$. Differentiating the Lyapunov functional $E_{\epsilon}(t)$ along the trajectory of $(2.1)$ leads to following identity for a.e. $t>0$ :

$$
\dot{E}_{\epsilon}(t)=-w_{t}(1, t) F\left(w_{t}(1, t)\right)+\epsilon\left[F^{2}\left(w_{t}(1, t)\right)+w_{t}^{2}(1, t)\right]-2 \epsilon E(t) .
$$


We claim that $\lim _{t \rightarrow \infty} w_{t}(1, t)=0$. By the claim, given the constant $M_{1}$ in (1.3) there exists some time $T_{1}>0$ such that

$$
\left|w_{t}(1, t)\right|<M_{1} \quad \forall t \geq T_{1} .
$$

By (2.10), (1.3), and (2.12) we get easily the following:

$$
\dot{E}_{\epsilon}(t) \leq-\left[C_{1}-\epsilon\left(1+C_{2}^{2}\right)\right] w_{t}^{2}(1, t)-2 \epsilon E(t) \forall t \geq T_{1} .
$$

Since $\epsilon$ satisfies the condition (2.9), it follows from (2.13) that

$$
\dot{E}_{\epsilon}(t) \leq-2 \epsilon E(t) \forall t \geq T_{1} .
$$

Using (2.14) and (2.10) we can get

$$
E(t) \leq 3 e^{-\frac{4 \epsilon\left(t-T_{1}\right)}{3}} E\left(T_{1}\right) \forall t \geq T_{1} .
$$

Thanks to decreasing property of energy $E(t)$ the inequality (2.15) holds $\forall t \geq 0$ :

$$
E(t) \leq 3 \exp \left\{\frac{4 \epsilon T_{1}}{3}\right\} \exp \left\{\frac{-4 \epsilon t}{3}\right\} E(0) \forall t \geq 0 .
$$

Hence the trajectory decays exponentially to zero in $X$ as $t \rightarrow \infty$.

To finish the proof we need to prove the claim $\lim _{t \rightarrow \infty} w_{t}(1, t)=0$. Denote by $L$ the linear functional $L: X \rightarrow \mathbb{R}$ such that $L f=f_{1}(1) \quad \forall f \in X$. Since $f_{1} \in$ $H_{L}^{1}(0,1), L$ is a bounded linear functional on $X$. One can write $w_{t}(1, t)=-L \tilde{A} \phi(t)$. For each $\phi^{0} \in \mathcal{D}(\tilde{A}),\|\tilde{A} \phi(t)\|$ being decreasing with $t$ (see [3, Theorem 3.1, p. 54] [2, Theorem 1.2, p. 102] and its proof, or [23, Corollary 3.11, p. 231]), we have $\|\tilde{A} \phi(t)\| \leq\left\|\tilde{A} \phi^{0}\right\|$. Therefore, it holds that

$$
\left|w_{t}(1, t)\right| \leq\|L\|\left\|\tilde{A} \phi^{0}\right\|
$$

From the equality

$$
w_{t}(1, t) F\left(w_{t}(1, t)\right)=\langle\tilde{A} \phi(t), \phi(t)\rangle_{X},
$$

we see that $w_{t}(1, t) F\left(w_{t}(1, t)\right)$ is bounded and tends to zero as $t \rightarrow \infty$, since $\lim _{t \rightarrow \infty}\|\phi(t)\|_{X}=0$ by Theorem 1. By (1.3) and the monotonicity of $F$, there exists some $T_{2}>0$ such that

$$
C_{1}\left|w_{t}(1, t)\right|^{2} \leq w_{t}(1, t) F\left(w_{t}(1, t)\right) \leq C_{2}\left|w_{t}(1, t)\right|^{2} \forall t \geq T_{2} .
$$

Hence $\lim _{t \rightarrow \infty} w_{t}(1, t)=0$, and the claim is proved. The proof is complete.

Remark 1. For uniform exponential stabilization the feedback control law must have a linear growth rate not only around zero but also at infinity (see Chen [4] for linear feedback and Zuazua [27] for nonlinear feedback). As proved in [26], with the saturated feedback control law in (1.4) the nonuniform exponential decay is the best decay rate to be expected. Here the (nonuniform) exponential decay of the smooth solutions is proved for the more general case (1.3).

2.2. High-dimensional wave equation. In this subsection we study the saturated nonlinear feedback stabilization of the wave equation in spatial higher dimension. We want to know whether or not, by means of saturated velocity feedback Neumann boundary control, the exponential decay property of the smooth solutions in the $1 \mathrm{D}$ case remains true for the higher dimensions.

Let $\Omega$ be a bounded, open, and connected set in $\mathbb{R}^{n}(n \leq 3)$ having a boundary $\Gamma=\partial \Omega$ of class $C^{2}$. For a fixed $x^{0} \in \mathbb{R}^{n}$ we set $m(x)=x-x^{0}$, and we consider the following partition of the boundary $\Gamma=\Gamma_{+} \cup \Gamma_{0}$ :

$$
\Gamma_{+}=\{x \in \Gamma \mid m(x) \cdot \nu(x)>0\}, \quad \Gamma_{0}=\{x \in \Gamma \mid m(x) \cdot \nu(x) \leq 0\}=\Gamma \backslash \Gamma_{+},
$$


where $\nu(x)$ is the unit normal vector at $x \in \Gamma$ pointing toward the exterior of $\Omega$, and "." denotes the scalar product in $\mathbb{R}^{n}$. Let $\partial_{\nu}$ and $\Delta$ denote the outward normal derivative and the Laplacian operator, respectively. Though $\Gamma_{+} \cap \Gamma_{0}=\varnothing$, we do not need to assume that $\overline{\Gamma_{+}} \cap \overline{\Gamma_{0}}=\varnothing$, but we do assume that $\operatorname{int}\left(\Gamma_{0}\right) \neq \varnothing$ and $\Gamma_{+} \neq \varnothing$. $\left(\overline{\Gamma_{+}}\right.$denotes the closure of $\left.\Gamma_{+}.\right)$

Consider the following wave equation controlled by the saturated feedback law $F$ :

$$
\begin{cases}w_{t t}(x, t)=\Delta w(x, t) & \forall(x, t) \in \Omega \times \mathbb{R}^{+}, \\ w(x, t)=0 & \forall(x, t) \in \Gamma_{0} \times \mathbb{R}^{+}, \\ \partial_{\nu} w(x, t)=-[m(x) \cdot \nu(x)] F\left(w_{t}\right) & \forall(x, t) \in \Gamma_{+} \times \mathbb{R}^{+}, \\ w(x, 0)=w^{0} \in H_{\Gamma_{0}}^{1}(\Omega), w_{t}(x, 0)=w^{1} \in L^{2}(\Omega), & \end{cases}
$$

where $H_{\Gamma_{0}}^{1}(\Omega)=\left\{f \in H^{1}(\Omega) \mid f=0\right.$ on $\left.\Gamma_{0}\right\}$ and $F: \mathbb{R} \rightarrow \mathbb{R}$ is defined as (1.3). The state space for $(2.18)$ is the Hilbert space $X=H_{\Gamma_{0}}^{1}(\Omega) \times L^{2}(\Omega)$ equipped with the inner product

$$
\langle f, g\rangle_{X}=\int_{\Omega}\left\{\nabla f_{1}(x) \cdot \nabla g_{1}(x)+f_{2}(x) g_{2}(x)\right\} d x .
$$

We define the nonlinear operator $W$ in $X$ by (2.19)

$$
\begin{aligned}
& W\left(f_{1}, f_{2}\right)=\left(-f_{2},-\Delta f_{1}\right), \\
& \mathcal{D}(W)=\left\{\left(f_{1}, f_{2}\right) \in\left[H_{\Gamma_{0}}^{1}(\Omega)\right]^{2} \mid \Delta f_{1} \in L^{2}(\Omega), \partial_{\nu} f_{1}=-m \cdot \nu F\left(f_{2}\right) \text { on } \Gamma_{+}\right\} .
\end{aligned}
$$

Notice that, in general, $\mathcal{D}(W) \not \subset\left[H^{2}(\Omega) \cap H_{\Gamma_{0}}^{1}(\Omega)\right] \times H_{\Gamma_{0}}^{1}(\Omega)$. Hence $\mathcal{D}(W)$ may contain elements $\left(f_{1}, f_{2}\right) \in\left(H_{\Gamma_{0}}^{1}(\Omega)\right)^{2}$ such that $f_{1} \notin H^{2}(\Omega)$. The Neumann boundary condition should be understood in the following sense: for each $\left(f_{1}, f_{2}\right) \in \mathcal{D}(W)$, $\partial_{\nu} f_{1}=-m \cdot \nu F\left(f_{2}\right)$ on $\Gamma_{+}$is satisfied if and only if the following holds:

$$
\int_{\Omega} \Delta f_{1}(x) \varphi(x) d x+\int_{\Omega} \nabla f_{1}(x) \cdot \nabla \varphi(x) d x=-\int_{\Gamma_{+}} m \cdot \nu F\left(f_{2}(x)\right) \varphi(x) d \Gamma \forall \varphi \in H_{\Gamma_{0}}^{1}(\Omega),
$$

where $d \Gamma$ denotes the surface measure associated to the boundary $\Gamma$. It is wellknown that $W$ is a maximal monotone operator on $X, \mathcal{D}(W)$ is dense in $X$, and the resolvent operators of $W$ are compact (see [22] and [25]). Then the system (2.18) can be written into the form

$$
\begin{aligned}
& \left(\dot{\phi}_{1}(t), \dot{\phi}_{2}(t)\right)+W\left(\phi_{1}(t), \phi_{2}(t)\right)=0, t>0, \\
& \left(\phi_{1}(0), \phi_{2}(0)\right)=\left(w^{0}, w^{1}\right),
\end{aligned}
$$

where $\phi_{1}(t)=w(\cdot, t)$ and $\phi_{2}(t)=w_{t}(\cdot, t)$. Thus $-W$ generates a semigroup of nonlinear contractions $\mathbb{G}(t)$ on $X$. If $\left(w^{0}, w^{1}\right) \in \mathcal{D}(W)$, then the solution $\phi(t)=$ $\mathbb{G}(t)\left(w^{0}, w^{1}\right) \in \mathcal{D}(W), \phi \in C([0, \infty), X)$ and $\dot{\phi} \in L^{\infty}([0, \infty), X), \dot{\phi}(t)+W \phi(t)=0$ for a.e. $t>0$, and $\|W \phi(t)\|_{X} \leq\left\|W\left(w^{0}, w^{1}\right)\right\|_{X} \forall t \geq 0$.

The following theorem gives a description of the long-time asymptotic behavior to the system (2.18).

Theorem 3. Assume that $x^{0} \in \mathbb{R}^{n}$ is such that $\operatorname{int}\left(\Gamma_{0}\right) \neq \varnothing$. Let $F: \mathbb{R} \rightarrow \mathbb{R}$ be continuous and increasing such that $F(0)=0$ and $F(s) s>0 \quad \forall s \neq 0$, and let the condition (1.3) be satisfied. Then the following statements are true:

(1) If $n=2$, for every initial condition $\left(w^{0}, w^{1}\right) \in \mathcal{D}(W)$ the solution $\left(w, w_{t}\right)$ of (2.18) decays like $t^{-2 / \alpha}$ to zero in $X$ as $t \rightarrow \infty$ for any $\alpha>0$.

(2) If $n=3$, for every initial condition $\left(w^{0}, w^{1}\right) \in \mathcal{D}(W)$ the solution $\left(w, w_{t}\right)$ of (2.18) decays like $t^{-2}$ to zero in $X$ as $t \rightarrow \infty$. 
The proof of Theorem 3 is technical and so postponed to Appendix A.

Remark 2. In the $2 \mathrm{D}$ case every smooth solution decays to zero faster than any polynomial $(1 / t)^{\alpha} \forall \alpha>0$, as $t \rightarrow \infty$. In the 3D case the decay rate is like $(1 / t)^{2}$. If $\overline{\Gamma_{+}} \cap \overline{\Gamma_{0}}=\varnothing$, then Theorem 3 is valid for all $n \geq 1$. For a more general case the reader is referred to $[17,15]$.

Such a result shows that, under the saturated velocity feedback Neumann boundary control, decaying property of the smooth solutions for the closed-loop system has a great change with spatial dimensional change.

3. Asymptotic rejection of boundary disturbance. In the previous section we proved that the saturated output feedback control stabilizes asymptotically the wave system at the equilibrium state $(0,0)$. In this section we shall study the robustness of the closed-loop system. In what follows, the state space always is $X=H_{L}^{1}(0,1) \times L^{2}[0,1]$.

The robustness of the closed-loop system depends on where the disturbance enters the system. It also depends on the type of stability of the the closed-loop system (see an interesting case in [19]). For example, if we consider an internal disturbance $f \in L^{1}((0, \infty), X)$ as follows

$$
\dot{\phi}+\tilde{A} \phi=f, \quad \phi(0)=\phi^{0},
$$

we can prove easily that every trajectory converges to zero as $t \rightarrow \infty$. Here we are interested in what happens to the boundary disturbances. In particular we consider the matched disturbance and control, i.e.,

$$
\left\{\begin{array}{l}
w_{t t}(x, t)=w_{x x}(x, t), \quad x \in(0,1), \quad t>0, \\
w(0, t)=0, \quad w_{x}(1, t)=-F\left(w_{t}(1, t)\right)+d(t), \quad t>0, \\
w(x, 0)=w_{0}(x), \quad w_{t}(x, 0)=w_{1}(x), \quad x \in(0,1) .
\end{array}\right.
$$

We will show that if the disturbance is smooth, the system (3.2) has a unique solution in $C([0, \infty), X)$. In particular, if the disturbance $d$ belongs to the Sobolev space $W^{1,1}(0, \infty)$ (i.e., $d$ and $d^{\prime} \in L^{1}(0, \infty)$ ), the system (3.2) has a unique solution in $C([0, \infty), X)$. Notice that $\lim _{t \rightarrow \infty} d(t)=0 \forall d \in W^{1,1}(0, \infty)$. We set $\mathbb{R}_{+}=(0, \infty)$ and $W^{n, 1}\left(\mathbb{R}_{+}\right)=\left\{d \in L^{1}\left(\mathbb{R}_{+}\right) \mid d^{\prime}, \ldots, d^{(n)} \in L^{1}\left(\mathbb{R}_{+}\right)\right\}$(see [1]).

The main result of the section is stated as follows.

Theorem 4. Let $d \in W^{1,1}\left(\mathbb{R}_{+}\right)$and let $\left(w_{0}, w_{1}\right) \in X$. Then the system (3.2) has a unique weak solution $\left(w(\cdot, t), w_{t}(\cdot, t)\right)$ that decays to zero in $X$ as $t \rightarrow \infty$.

Because of the presence of the disturbance, the system (3.2) is a nonautonomous system. Before proving Theorem 4, let us recall next the weak solutions and the strong solutions to the system (3.2).

Definition 1. Let $T>0$. A continuous function $w(\cdot, t)$ from $[0, T]$ to $H_{L}^{1}(0,1)$ is called strong solution to the system (3.2) on $[0, T]$ if it is continuously differentiable from $[0, T]$ to $L^{2}(0,1)$ and if $\left(w(\cdot, t), w_{t}(\cdot, t)\right)$ is absolutely continuous from every compact interval of $(0, T)$ to $X$ with $\left(w(\cdot, t), w_{t}(\cdot, t)\right) \in H^{2}(0,1) \times H_{L}^{1}(0,1) \forall t \in[0, T]$, and $w_{x}(1, t)=-F\left(w_{t}(1, t)\right)+d(t)$ such that the PDE $(3.2)$ is satisfied in $L^{2}(0,1)$ at a.e. $t \in(0, T)$.

TheOrem 5. Let $d \in W^{2,1}\left(\mathbb{R}_{+}\right)$, and let $\left(w_{0}, w_{1}\right) \in X \cap\left(H^{2}(0,1) \times H_{L}^{1}(0,1)\right)$ such that the compatibility condition $w_{0}^{\prime}(1)=-F\left(w_{1}(1)\right)+d(0)$ is satisfied. Then the system (3.2) has a unique strong solution $\varphi(t)=\left(w(\cdot, t), w_{t}(\cdot, t)\right) \in C([0, \infty) ; X)$. 
Moreover the strong solution continuously depends on the initial condition and the input signal as follows:

$$
\|\varphi(t)-\tilde{\varphi}(t)\|_{X} \leq\|\varphi(s)-\tilde{\varphi}(s)\|_{X}+|d(s)-\tilde{d}(s)|+|d(t)-\tilde{d}(t)|+\int_{s}^{t}\left|d^{\prime}(\tau)-\tilde{d}^{\prime}(\tau)\right| d \tau,
$$

where $\tilde{\varphi}(t)$ is the strong solution corresponding to $\left(\tilde{w}_{0}, \tilde{w}_{1}\right)$ and $\tilde{d}$, and $0 \leq s \leq t<\infty$.

Proof. Let $\varphi(x, t)=\left(w(x, t), w_{t}(x, t)\right)^{\top}$. Consider the affine state transformation in $X$ :

$$
\phi(x, t)=\varphi(x, t)-\left(\begin{array}{l}
x \\
0
\end{array}\right) d(t) .
$$

Obviously $\varphi(x, t)$ is a strong solution to the PDE (3.2) if and only if $\phi(x, t)$ is a strong solution to the following PDE :

$$
\begin{gathered}
\left\{\begin{array}{l}
\phi_{1 t}(x, t)=\phi_{2}(x, t)-x d^{\prime}(t), \\
\phi_{2 t}(x, t)=\phi_{1 x x}(x, t),
\end{array}\right. \\
\phi_{1}(0, t)=0, \quad \phi_{1 x}(1, t)=-F\left(\phi_{2}(1, t)\right), \\
\phi_{1}(x, 0)=\phi_{1}^{0}(x)=w_{0}(x)-x d(0), \quad \phi_{2}(x, 0)=\phi_{2}^{0}(x)=w_{1}(x) .
\end{gathered}
$$

Therefore, equivalently we prove existence and uniqueness of the strong solution to the system (3.5)-(3.7). Let us set $f(x, t)=(-x, 0)^{\top} d^{\prime}(t)$. Then the system is written as an evolution equation of the form (3.1).

By the assumptions we have $f \in W^{1,1}\left(\mathbb{R}_{+}\right)$and $\phi(0) \in \mathcal{D}(\tilde{A})$. The PDE (3.5)(3.7) or (3.1) has a unique strong solution $\phi(t)$ as defined as in Definition 1 (cf. the existence theorem of [10] or [3, p. 64]). If $\tilde{\phi}(t)$ is a strong solution of (3.1) corresponding to $(\tilde{\phi}(0), \tilde{f})$, it is well known that the following continuous dependence condition is satisfied:

$$
\|\phi(t)-\tilde{\phi}(t)\|_{X} \leq\|\phi(s)-\tilde{\phi}(s)\|_{X}+\int_{s}^{t}\|f(\tau)-\tilde{f}(\tau)\|_{X} d \tau \quad \forall 0 \leq s \leq t .
$$

Hence the required inequality (3.3) is easily established by using (3.8), $f$, and the transformation (3.4). The proof of Theorem 5 is complete.

In particular the following estimate holds for the system (3.5)-(3.7):

$$
\|\phi(t)-\tilde{\phi}(t)\|_{X} \leq\left\|\phi^{0}-\tilde{\phi}^{0}\right\|_{X}+\int_{0}^{t}\left|d^{\prime}(\tau)-\tilde{d}^{\prime}(\tau)\right| d \tau \forall \forall t \geq 0 .
$$

Definition 2. By (3.9) the nonlinear mapping $\Lambda$, which to each $\left(\phi^{0}, d\right) \in \mathcal{D}(\tilde{A}) \times$ $W^{2,1}\left(\mathbb{R}_{+}\right)$associates the strong solution of $(3.5)-(3.7), \Lambda\left(\phi^{0}, d\right)(t)=\phi(t)$, is continuous from $X \times W^{1,1}\left(\mathbb{R}_{+}\right)$to $C([0, T] ; X)$. It admits a unique continuous extension on the whole space $X \times W^{1,1}\left(\mathbb{R}_{+}\right)$. For each $\left(\phi^{0}, d\right) \in X \times W^{1,1}\left(\mathbb{R}_{+}\right), \phi(t)=\Lambda\left(\phi^{0}, d\right)(t)$ is called a weak solution of (3.5)-(3.7). Through the transformation $(3.4), \varphi(t)$ is called a weak solution of (3.2).

By density of $\mathcal{D}(\tilde{A}) \times W^{2,1}\left(\mathbb{R}_{+}\right)$in $X \times W^{1,1}\left(\mathbb{R}_{+}\right)$, the following is true.

Copyright $@$ by SIAM. Unauthorized reproduction of this article is prohibited. 
Corollary 3.1. For each $\left(\phi^{0}, d\right) \in X \times W^{1,1}\left(\mathbb{R}_{+}\right)$, the system (3.5)-(3.7) or (3.2) has a unique weak solution.

Remark 3. By density of $\mathcal{D}(\tilde{A}) \times W^{2,1}\left(\mathbb{R}_{+}\right)$in $X \times W^{1,1}\left(\mathbb{R}_{+}\right)$and the continuity (3.9) it suffices to prove Theorem 4 for strong solutions.

Proof of Theorem 4. Let $\left(\phi^{0}, d\right) \in \mathcal{D}(\tilde{A}) \times W^{2,1}\left(\mathbb{R}_{+}\right)$, and let us consider the system (3.5)-(3.7) on the extended state space $Z=X \times W^{1,1}\left(\mathbb{R}_{+}\right)$. The state space $Z$ is a Banach space equipped with the norm

$$
\left\|\left(\begin{array}{c}
\phi_{1} \\
\phi_{2} \\
d
\end{array}\right)\right\|_{Z}=\left\|\left(\begin{array}{c}
\phi_{1} \\
\phi_{2}
\end{array}\right)\right\|_{X}+\int_{0}^{\infty}\left(|d(t)|+\left|d^{\prime}(t)\right|\right) d t .
$$

The semigroup $(\mathbb{Z}(t))_{t \geq 0}$ on $Z$ is defined by the solution of (3.5)-(3.7) as follows:

$$
\mathbb{Z}(t)\left(\phi^{0}, d\right)=(\phi(t), d(t+\cdot))=\left(\Lambda\left(\phi^{0}, d\right)(t), d(t+\cdot)\right) .
$$

By (3.9), $(\mathbb{Z}(t))_{t \geq 0}$ is a semigroup of nonlinear contractions on $Z$. Since $\| \mathbb{Z}(t+s)$ $\left(\phi^{0}, d\right)\left\|_{Z} \leq\right\| \mathbb{Z}(t)\left(\phi^{0}, d\right) \|_{Z} \forall t, s \geq 0$, the limit exists: $\lim _{t \rightarrow \infty}\|(\phi(t), d(t+\cdot))\|_{Z}=c$ for some real number $c \geq 0$. Immediately, we have

$$
\lim _{t \rightarrow \infty}\|d(t+\cdot)\|_{W^{1,1}\left(\mathbb{R}_{+}\right)}=0
$$

As the resolvent operator of $\tilde{A}$ is compact, the orbit $\gamma\left(\phi^{0}, d\right)$ is precompact (cf. [10]):

$$
\gamma\left(\phi^{0}, d\right)=\left\{\mathbb{Z}(t)\left(\begin{array}{c}
\phi^{0} \\
d
\end{array}\right) \mid t \in \mathbb{R}_{+}\right\} .
$$

Hence the $\omega$-limit set $\omega\left(\phi^{0}, d\right)$ is nonempty. It is clear that

$$
\omega\left(\phi^{0}, d\right)=\left\{(\varphi, 0) \mid \exists\left(t_{n}\right) \text { such that } \lim _{n \rightarrow \infty} t_{n}=\infty, \quad \varphi=\lim _{n \rightarrow \infty} \phi\left(t_{n}\right)\right\} .
$$

We claim that the $\omega$-limit set is reduced to $(0,0)$. By the Theorem 1.1.8 in Haraux (p. 12 of [14]) it follows that $\lim _{t \rightarrow \infty} \phi(t)=0$. By transformation (3.4) and $\lim _{t \rightarrow \infty}$ $d(t)=0$,

$$
\lim _{t \rightarrow \infty}\left(w(\cdot, t), w_{t}(\cdot, t)\right)=0 .
$$

Now it is sufficient to prove the claim. Take $(\varphi, 0) \in \omega\left(\phi^{0}, d\right)$. By the LaSalle invariance principle $\mathbb{Z}(t)(\varphi, 0) \in \omega\left(\phi^{0}, d\right)$. As $(\varphi, 0) \in \mathcal{D}(\tilde{A}) \times\{0\},(\phi(t), 0)=\mathbb{Z}(t)(\varphi, 0)$ is a strong solution of (3.5)-(3.7). Hence $\phi_{2}(1, t)=0 \forall t \geq 0$, as the energy is constant along the trajectory. By the same argument as for the proof of Theorem 1, it is proved that $\varphi=0$. Therefore the $\omega$-limit set is reduced to $(0,0)$. So the claim is proved and the proof of Theorem 4 is complete.

Remark 4. By direct computation the generator $\mathbb{A}$ of the $C_{0}$ semigroup $(\mathbb{Z}(t))_{t \geq 0}$ is given by

$$
\mathcal{D}(\mathbb{A})=\mathcal{D}(\tilde{A}) \times \times W^{2,1}\left(\mathbb{R}_{+}\right), \quad \mathbb{A}(\phi, d)=\left(-\tilde{A} \phi+B d^{\prime}(0), d^{\prime}\right) \quad \forall(\phi, d) \in \mathcal{D}(\mathbb{A}),
$$

where $B=(-x, 0)^{\top}$ and $\tilde{A}$ is defined in $(2.2)-(2.3)$. The resolvent operator $(I-\lambda \mathbb{A})^{-1}$ is not compact for $\lambda>0$. However the resolvent operator $(I+\lambda \tilde{A})^{-1}$ is compact for any $\lambda>0$. By similar arguments as in [10], one proves that the orbit $\gamma\left(\phi^{0}, d\right)$ is precompact. 
4. Sliding mode control as limit of saturated feedback laws. Let $M$ be a positive constant. We consider the specific control law $u(t)=-F_{\lambda}\left(w_{t}(1, t)\right), \lambda>0$ for (1.2), where $F_{\lambda}$ is defined by (1.5). Note that $\operatorname{sign}(x)$ is a multivalued function. $F_{\lambda}(x)$ is the Yosida approximation of $M \operatorname{sign}(x): \lim _{\lambda \rightarrow 0^{+}} F_{\lambda}(x)=M \operatorname{sign}^{0}(x)$, where $\operatorname{sign}^{0}(x)$ is the element $y \in \operatorname{sign}(x)$ of the least norm. Here we have $\operatorname{sign}^{0}(0)=0$ and $\operatorname{sign}^{0}(x)=\operatorname{sign}(x) \forall x \neq 0$. In this section we shall discuss the stabilizability of the wave equation by two feedback control laws.

First we consider the closed-loop system governed by

$$
\left\{\begin{array}{l}
w_{t t}(x, t)=w_{x x}(x, t), \quad x \in(0,1), \quad t>0 \\
w(0, t)=0, \quad w_{x}(1, t)=-F_{\lambda}\left(w_{t}(1, t)\right), \quad t>0, \\
w(x, 0)=w_{0}(x), \quad w_{t}(x, 0)=w_{1}(x), \quad x \in(0,1) .
\end{array}\right.
$$

According to Theorem 1 the closed-loop system (4.1) is globally asymptotically stable for any $\lambda>0$.

Secondly, as a limit case of the Yosida approximation, we consider the sliding mode control law, i.e.,

$$
\left\{\begin{array}{l}
w_{t t}(x, t)=w_{x x}(x, t), \quad x \in(0,1), \quad t>0 \\
w(0, t)=0, \quad w_{x}(1, t) \in-M \operatorname{sign}\left(w_{t}(1, t)\right), \quad t>0 \\
w(x, 0)=w_{0}(x), \quad w_{t}(x, 0)=w_{1}(x), \quad x \in(0,1) .
\end{array}\right.
$$

We want to know whether or not the closed-loop system (4.2) has asymptotic stability.

Contrary to the intuitive feeling the situation is not so simple. To guarantee existence and uniqueness of physically reasonable solutions to the system (4.2) the boundary condition is understood as

$$
w_{x}(1, t) \in-M \operatorname{sign}\left(w_{t}(1, t)\right) .
$$

Equation (4.3) is said to be satisfied if the left-hand member evaluated at time $t$ has value in the subset defined by the right-hand member.

To study asymptotic stability of the system (4.2) we first prove existence and uniqueness of its solutions in the energy space $X=H_{L}^{1}(0,1) \times L^{2}(0,1)$. Then some required regularity of the trajectories will be established for the purpose of asymptotic analysis.

Define the nonlinear operator $A: \mathcal{D}(A) \rightarrow X$ by

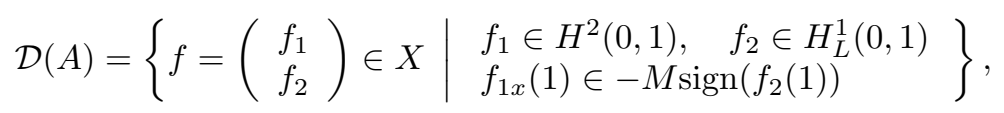

and, for each $f \in \mathcal{D}(A)$,

$$
A f=\left(\begin{array}{c}
-f_{2} \\
-f_{1 x x}
\end{array}\right)
$$

Above $f_{1 x}(x)$ or $\partial_{x} f_{1}(x)$ denotes the first order derivative of $f_{1}(x)$ w.r.t. $x$. Notice that $H^{n}(0,1)=\left\{f \mid f, f^{\prime}, \ldots, f^{(n)} \in L^{2}(0,1)\right\}$ where $f^{\prime}$ and $f^{(n)}$ denote the derivatives of first order and $n$th order, respectively, $\forall n \in \mathbb{N}$.

We set $\phi_{1}(t)=w(\cdot, t)$ and $\phi_{2}(t)=w_{t}(\cdot, t)$ in what follows. We use the notation $\phi(t)=\left(\phi_{1}(t), \phi_{2}(t)\right)^{T}$ whenever there is no confusion. Then the closed-loop system $(4.2)$ is written formally as

$$
\left\{\begin{array}{l}
\dot{\phi}(t)+A \phi(t)=0 \\
\phi(0)=\phi^{0} \in X
\end{array}\right.
$$

Copyright $@$ by SIAM. Unauthorized reproduction of this article is prohibited. 
Lemma 2. The operator $A$ defined by (4.4)-(4.5) is maximal monotone on $X$, $\mathcal{D}(A)$ is dense in $X$, and the resolvent operator $(I+\lambda A)^{-1}$ is compact $\forall \lambda>0$.

Proof. The proof is similar to that of Lemma 1 and so omitted. Remark only that $A$ is monotone maximal if and only if $\operatorname{sign}(x)$ is monotone maximal from $\mathbb{R}$ to $\mathbb{R}$. The function $\operatorname{sign}(x)$ is clearly monotone. It is also maximal because $(I+\operatorname{sign})$ is onto. Indeed,

$$
(I+\operatorname{sign})^{-1}(x)=\left\{\begin{array}{lr}
x-1, & x>1, \\
0, & x \in[-1,1], \\
x+1, & x<-1 .
\end{array}\right.
$$

So the proof of Lemma 2 is complete.

The following result can be proved by applying the classical theory of nonlinear semigroups (see [3] or [10]).

Lemma 3. The operator $(-A)$ generates a nonlinear semigroup $(\mathbb{S}(t))_{t \geq 0}$ of contractions on $X$. The nonlinear system (4.6) has a unique solution in $C([0, \infty), X)$ given by $\phi(t)=\mathbb{S}(t) \phi^{0}$. For every $\phi^{0} \in \mathcal{D}(A)$, the solution $\phi(t) \in \mathcal{D}(A) \forall t \geq 0 ; \phi(t)$ is strongly differentiable at a.e. $t>0 ; \dot{\phi} \in L^{\infty}((0, \infty), X)$; and the equation (4.6) is satisfied at a.e. $t>0$.

Direct computations give all equilibrium states of the system (4.6).

Lemma 4. The set of all equilibrium states for the system (4.6) is given by

$$
\Gamma_{e}=\left\{\left(\begin{array}{c}
c x \\
0
\end{array}\right) \in X \mid c \in[-M, M]\right\} .
$$

Moreover each state in $\Gamma_{e}$ is Lyapunov stable.

Proof. Solving the equation $A \phi=0$ with $\phi \in \mathcal{D}(A)$ gives us $\phi_{1}=c x$ and $\phi_{2}=0$. The boundary condition $\phi_{1 x}(1) \in M \operatorname{sign}(0)$ implies that $c \in[-M, M]$.

Let $\phi^{e} \in \Gamma_{e}$. Since the semigroup is contractive, we have $\left\|\mathbb{S}(t) \phi^{0}-\phi^{e}\right\|_{X} \leq$ $\left\|\phi^{0}-\phi^{e}\right\|_{X} \forall t \geq 0$. Hence $\phi^{e}$ is Lyapunov stable.

Let $\gamma\left(\phi^{0}\right)$ be the orbit through $\phi^{0} \in X$ such that $\gamma\left(\phi^{0}\right)=\left\{\mathbb{S}(t) \phi^{0} \mid t \geq 0\right\}$. Let the $\omega$-limit set $\omega\left(\phi^{0}\right)$ be defined by

$$
\omega\left(\phi^{0}\right)=\left\{\varphi \mid \exists\left(t_{n}\right) \text { such that } \lim _{n \rightarrow \infty} t_{n}=\infty, \quad \varphi=\lim _{n \rightarrow \infty} \mathbb{S}\left(t_{n}\right) \phi^{0}\right\} .
$$

The long-time asymptotic behavior of the trajectory $\phi(t)=\mathbb{S}(t) \phi^{0}$ is described by the following theorem.

TheOREm 6. (i) For each $\phi^{0} \in X$, the orbit $\gamma\left(\phi^{0}\right)$ of the system (4.6) is precompact. (ii) For each $\phi^{0} \in X$, the $\omega$-limit set $\omega\left(\phi^{0}\right)$ is nonempty, compact, and connected in $X$; it is positively invariant under the semigroup $\left(\mathbb{S}\left(t_{n}\right)\right)_{t \geq 0}$, and the following property holds:

$$
\lim _{t \rightarrow \infty} \min _{y \in \omega\left(\phi^{0}\right)}\left\|\mathbb{S}\left(t_{n}\right) \phi^{0}-y\right\|_{X}=0 .
$$

(iii) For each $\phi^{0} \in \mathcal{D}(A)$, the corresponding trajectory $\phi(t)$ converges to a periodic trajectory noted $\phi_{p}(t)$ or to an equilibrium point of $\Gamma_{e}$, as $t \rightarrow \infty$; moreover the periodic trajectory $\phi_{p}(t)$ has period equal to 2 and constant energy $\left\|\phi_{p}(t)\right\|_{X}^{2}=\mu^{2} \leq$ $M^{2}, M$ the feedback gain. 
Proof. By compactness of $(I+A)^{-1}$ and similar arguments of [10], the assertions (i)-(ii) are proved (see [14]).

(iii) Let us analyze the asymptotic behavior of the trajectory through $\phi^{0} \in \mathcal{D}(A)$. Let $V(\phi)=\|\phi\|_{X}^{2}$. We have $\phi(t) \in \mathcal{D}(A)$ and a.e. differentiable $\forall t \geq 0$. Differentiating $V(\phi(t))$ along the trajectory gives us

$$
\dot{V}(\phi(t))=-2\langle A \phi(t), \phi(t)\rangle_{X}=-2 M\left|\phi_{2}(1, t)\right| \leq 0, \text { a.e. } t>0 .
$$

The function $t \mapsto V(\phi(t))$ is decreasing, and hence $\lim _{t \rightarrow \infty} V(\phi(t))=\mu^{2} \geq 0$. As the orbit is precompact, the $\omega$-limit set is nonempty and $\omega\left(\phi^{0}\right) \subset \mathcal{D}(A) \cap E_{\mu}$ (see $[10$, Theorem 5] or [9, Lemma 2.3]), where

$$
E_{\mu}=\left\{y \in \mathcal{D}(A) \mid\|y\|_{X}=\mu, y_{2}(1)=0\right\} .
$$

Take $\varphi \in \omega\left(\phi^{0}\right)$. We set $\left(w(x, t), w_{t}(x, t)\right)=(\mathbb{S}(t) \varphi)(x)$. By the LaSalle invariance principle $\mathbb{S}(t) \varphi \in \omega\left(\phi^{0}\right) \forall t \geq 0$. By Lemma 3 the following PDE is satisfied by $w(x, t)$ :

$$
\left\{\begin{array}{l}
w_{t t}(x, t)=w_{x x}(x, t) \quad \forall x \in(0,1), \quad t>0, \\
w(0, t)=0, w_{t}(1, t)=0, \quad w_{x}(1, t) \in-M \operatorname{sign}(0), \\
w(x, 0)=\varphi_{1}(x), \quad w_{t}(x, 0)=\varphi_{2}(x),
\end{array}\right.
$$

where $w_{t}(1, t)=0$ because $\|\mathbb{S}(t) \varphi\|=\|\varphi\|=\mu$. We know that the above PDE (4.8) has a unique solution, but we prove the solution to be periodic of period $T=2$.

Indeed, from $(4.8), w_{t}(1, t)=0$ implies that $w(1, t)$ is constant. The compatibility condition gives $w(1, t)=c=\varphi_{1}(1)$. We set $v(x, t)=w(x, t)-c x$. Clearly $w(x, t)$ satisfies the above PDE (4.8) if and only if $v(x, t)$ satisfies the following PDE

$$
\left\{\begin{array}{l}
v_{t t}(x, t)=v_{x x}(x, t) \quad \forall x \in(0,1), \quad t>0 \\
v(0, t)=0, \quad v(1, t)=0, \\
v(x, 0)=\varphi_{1}(x)-c x, \quad v_{t}(x, 0)=\varphi_{2}(x)
\end{array}\right.
$$

with the constraint $v_{x}(1, t)+c \in M \operatorname{sign}(0)$. The PDE (4.9) has a unique solution. It is easy to see that the eigenvalues and eigenfunctions of (4.9) are given by

$$
\lambda_{n}=i n \pi, \quad e_{n}(x)=\sqrt{2} \sin (n \pi x), \quad n \in \mathbb{N}^{*} .
$$

The family $\left\{e_{n}(x), n \in \mathbb{N}^{*}\right\}$ forms an orthonormal basis for $L^{2}(0,1)$. To solve (4.9) we write the initial condition as follows:

$$
\varphi_{1}(x)-\varphi_{1}(1) x=\sum_{n=1}^{\infty} a_{n} \sin (n \pi x), \quad \varphi_{2}(x)=\sum_{n=1}^{\infty} b_{n} \sin (n \pi x)
$$

where

$$
a_{n}=2 \int_{0}^{1}\left(\varphi_{1}(x)-c x\right) \sin (n \pi x) d x, \quad b_{n}=2 \int_{0}^{1} \varphi_{2}(x) \sin (n \pi x) d x .
$$

The solution to (4.9) is found to be

$$
v(x, t)=\sum_{n=1}^{\infty}\left[a_{n} \cos (n \pi t)+b_{n} \frac{\sin (n \pi t)}{n \pi}\right] \sin (n \pi x) .
$$

Copyright (c) by SIAM. Unauthorized reproduction of this article is prohibited. 
Then the solution to (4.8) is given by

$$
\begin{gathered}
w(x, t)=\varphi_{1}(1) x+\sum_{n=1}^{\infty}\left[a_{n} \cos (n \pi t)+b_{n} \frac{\sin (n \pi t)}{n \pi}\right] \sin (n \pi x), \\
w_{t}(x, t)=\sum_{n=1}^{\infty}\left[-n \pi a_{n} \sin (n \pi t)+b_{n} \cos (n \pi t)\right] \sin (n \pi x),
\end{gathered}
$$

and

$$
w_{x}(1, t)=\varphi_{1}(1)+\sum_{n=1}^{\infty}(-1)^{n}\left[n \pi a_{n} \cos (n \pi t)+b_{n} \sin (n \pi t)\right]
$$

Each function in (4.10)-(4.12) is a periodic function of time $t$ with period equal to 2. Moreover each series in (4.10)-(4.12) converges uniformly on $[0,1] \times[0,2]$. By uniqueness of the solution the constructed $\phi_{p}(t)=\left(w(\cdot, t), w_{t}(\cdot, t)\right)$ is the solution to (4.8). If $\phi^{0}(x)=(c x, 0), c \in \mathbb{R}$, then the trajectory converges to an equilibrium state in $\Gamma_{e}$, as $t \rightarrow \infty$.

As $\max _{t \in[0,2]}\left|w_{x}(1, t)\right| \leq M$, direct computations from (4.8) and (4.10)-(4.12) lead us to

$$
\|\varphi\|_{X}^{2}=\int_{0}^{1}\left(w_{x}^{2}(x, t)+w_{t}^{2}(x, t)\right) d x=\mu^{2}=\frac{1}{2} \int_{0}^{2} w_{x}^{2}(1, t) d t \leq M^{2} .
$$

Since the $\omega$-limit set $\omega\left(\phi^{0}\right)$ is connected, the trajectory through $\phi^{0}$ converges necessarily to one (and only one) periodic solution or one equilibrium state of the norm smaller than or equal to $M$. The proof of Theorem 6 is complete.

Remark 5. From Theorem 6 follow several important points:

(i) The closed-loop system with the sliding mode control is nonasymptotically stable. It is shown that the stability of the system (4.2) cannot be deduced from that of the system (4.1).

(ii) The system (4.2) may be taken as practically stabilized in the following sense: it is sufficient to make $M$ small for the system to be stabilized around the origin.

(iii) By the method of characteristics due to Greenberg and Li [11] we can check that the trajectory from the initial condition $\phi^{0}(x)=(2 M x, 0)$ converges to the equilibrium state $(0,0)$ in finite time. By using the same method, it can be shown that the solution of (4.6) from $\phi^{0}(x)=\left(\varphi_{1}(x), \varphi_{2}(x)\right) \in X$ is a periodic solution of period $T=2$ if $\phi^{0}(x)$ satisfies the condition: $\left\|\varphi_{1}^{\prime}+\varphi_{2}\right\|_{L^{\infty}} \leq M$ and $\left\|\varphi_{1}^{\prime}-\varphi_{2}\right\|_{L^{\infty}} \leq M$.

Similarly we can consider the matched disturbance and control:

$$
\left\{\begin{array}{l}
w_{t t}(x, t)=w_{x x}(x, t), \quad x \in(0,1), \quad t>0, \\
w(0, t)=0, \quad w_{x}(1, t) \in-M \operatorname{sign}\left(w_{t}(1, t)\right)+d(t), \\
w(x, 0)=w_{0}(x), \quad w_{t}(x, 0)=w_{1}(x), \quad x \in(0,1) .
\end{array}\right.
$$

Similar arguments allow us to prove the following result.

Theorem 7. Let $d \in W^{2,1}\left(\mathbb{R}_{+}\right)$, and let $\left(w_{0}, w_{1}\right) \in \mathcal{D}(A)$. Then the unique solution of $(4.13),\left(w(\cdot, t), w_{t}(\cdot, t)\right)$, converges to either a periodic solution $\phi_{p}(t)$ or an equilibrium state of $\Gamma_{e}$ in $X$, as $t \rightarrow \infty$. Moreover the periodic solution $\phi_{p}(t)$ lies on a sphere $\left\|\phi_{p}(t)\right\|_{X}=\mu \leq M$. 
5. Conclusions. In this paper we studied the stabilization problem of a wave equation by means of a priori bounded nonlinear feedback control laws. Different nonlinear feedback controllers have been designed to stabilize the system. Nonlinear semigroup theory has been applied to prove existence and uniqueness of solutions for the closed-loop system (with or without nonhomogeneous term on the boundary). The LaSalle invariance principle has been applied to study asymptotic stability or the long-time asymptotic behavior for the closed-loop system.

For the saturated output feedback control law, we established different decay rates of the smooth solutions by using appropriate Lyapunov functionals: in the 1D case the decay rate is exponential with time; in the $2 \mathrm{D}$ or $3 \mathrm{D}$ case the decay rate is polynomial. Moreover, in the 1D case, the energy of the closed-loop system decays to zero as $t \rightarrow \infty$, independently of unknown boundary disturbance in $W^{1,1}\left(\mathbb{R}_{+}\right)$. It is meant that the stabilized closed-loop system has robust asymptotic stability faced to the boundary disturbance.

As the Yosida approximation limit case of the saturated output feedback laws, we considered the sliding mode control law. According to Theorem 1 the closed-loop system with the saturated feedback control law is globally asymptotically stable for any $\lambda>0$. However the closed-loop system obtained by passing to limit as $\lambda \rightarrow 0$, i.e., the sliding mode control law, has no asymptotical stability: each trajectory of the closed-loop system is bounded and converges asymptotically to a periodic one whose energy is bounded by the feedback gain $M$.

Notice that our closed-loop system is governed by a nonlinear contraction semigroup. However, in practice we do not always have dynamical systems governed by contractive semigroups. For example, take the scalar system $\dot{x}(t) \in \operatorname{sign}(x(t))$ with $x(0)=0$. The system has three different solutions: $x(t)=0, x(t)=t$, and $x(t)=-t$. Of course the system is not contractive. If we take another system $\dot{x}(t) \in-\operatorname{sign}(x(t))$ with $x(0)=0$, the only solution is $x(t)=0$. To extend the sliding mode control to infinite-dimensional Hilbert spaces, it is essential to study existence and uniqueness of the associated solutions; that has been the motivation of our present work.

\section{Appendix A.}

As our proof is inspired by ingredients and reasoning in the literature (cf. [16, $27,15]$ ), only essential idea of proof is given here. Note that every solution decays asymptotically to zero in $X$ whatever is the initial condition $\left(w^{0}, w^{1}\right) \in X$ (see [27] or [17] for a proof). The following important technical lemma has been proved in [16] and is restated here for the reader's convenience. Let $(u, v)$ and $\|u\|$ denote the inner product and the induced norm in $L^{2}(\Omega)$, respectively: $(u, v)=\int_{\Omega} u(x) v(x) d x$ and $\|u\|^{2}=(u, u)$.

Lemma 5. Assume that $n \leq 3$, and let $(u, v) \in \mathcal{D}(W)$. Then the following inequality holds:

$$
2(\Delta u, m \cdot \nabla u) \leq(n-2)\|\nabla u\|^{2}+2 \int_{\Gamma} \partial_{\nu} u(m \cdot \nabla u) d \Gamma-\int_{\Gamma}(m \cdot \nu)|\nabla u|^{2} d \Gamma,
$$

where $d \Gamma$ is the surface measure associated to the boundary $\Gamma$.

Proof of Theorem 3. Let $\left(w^{0}, w^{1}\right) \in \mathcal{D}(W)$, and let $\left(w, w_{t}\right)$ be the trajectory from $\left(w^{0}, w^{1}\right)$. We set

$$
E(t)=\frac{1}{2} \int_{\Omega}\left\{|\nabla w|^{2}+\left|w_{t}\right|^{2}\right\} d x, \quad \rho(t)=\int_{\Omega}\left[2 w_{t} m \cdot \nabla w+(n-1) w_{t} w\right] d x .
$$


We consider the Lyapunov functional candidate as follows:

$$
E_{\epsilon}(t)=E(t)+\epsilon E^{\frac{p-1}{2}}(t) \rho(t), \quad \epsilon>0, p>1 .
$$

By differentiating w.r.t. time $E_{\epsilon}(t)$ along the trajectory we get

$$
\dot{E}_{\epsilon}(t)=\left[1+\frac{\epsilon(p-1)}{2} E^{\frac{p-3}{2}}(t) \rho(t)\right] \dot{E}(t)+\epsilon E^{\frac{p-1}{2}}(t) \dot{\rho}(t),
$$

where

$$
\dot{E}(t)=-\int_{\Gamma_{+}}(m \cdot \nu) F\left(w_{t}(x, t)\right) w_{t}(x, t) d \Gamma
$$

$\dot{\rho}(t)=2(\Delta w, m \cdot \nabla w)-(n-1)\|\nabla w\|^{2}-\left\|w_{t}\right\|^{2}+\int_{\Gamma_{+}}(m \cdot \nu)\left[\left|w_{t}\right|^{2}-(n-1) F\left(w_{t}\right) w\right] d \Gamma$.

We claim that there exists some positive constant $\epsilon>0$ such that the following holds:

(i) $\quad \frac{1}{2} E(t) \leq E_{\epsilon}(t) \leq \frac{3}{2} E(t) \forall t \geq 0$;

(ii) $\dot{E}_{\epsilon}(t) \leq-\epsilon\left(\frac{2}{3}\right)^{\frac{p+3}{2}} E_{\epsilon}^{\frac{p+1}{2}}(t) \quad \forall t \geq 0 \forall p>1$ if $n=2$ (and $\forall p \geq 2$ if $n=3$, respectively).

Note that $\epsilon$ may depend on the initial condition $\left(w^{0}, w^{1}\right)$. Set $\tilde{\epsilon}=\epsilon\left(\frac{2}{3}\right)^{\frac{p+3}{2}}$. From (i) and (ii) it follows that

$$
E(t) \leq 2 E_{\epsilon}(t) \leq \frac{2^{\frac{p+1}{p-1}} E_{\epsilon}(0)}{\left(2+\tilde{\epsilon}(p-1) E_{\epsilon}^{\frac{p-1}{2}}(0) t\right)^{\frac{2}{p-1}}} \forall t \geq 0, \forall p>1 \text { if } n \leq 2
$$

(and $\forall p \geq 2$ if $n=3$, respectively). The last inequality implies that the assertions (1) and (2) of Theorem 3 hold.

To complete the proof it is sufficient to prove the claim. By the Cauchy inequality and the Poincaré inequality there exists some constant $\alpha_{1}>0$ such that $|\rho(t)| \leq$ $\alpha_{1} E(t)$. Assume that $E(0)>0$, and notice that $E(t)$ is decreasing. By taking $0<\epsilon \leq \epsilon_{1}$ where

$$
\epsilon_{1}=\frac{1}{2 \alpha_{1} E^{\frac{p-1}{2}}(0)}
$$

the assertion (i) is proved.

Let us prove (ii):

$$
\forall \epsilon \leq \epsilon_{2}=\frac{1}{(p-1) \alpha_{1} E^{\frac{p-1}{2}}(0)}
$$

by (A.2) we have

$$
\dot{E}_{\epsilon}(t) \leq-\frac{1}{2} \int_{\Gamma_{+}}(m \cdot \nu) F\left(w_{t}\right) w_{t} d \Gamma+\epsilon E^{\frac{p-1}{2}}(t) \dot{\rho}(t)
$$

We set $R=\|m\|_{L^{\infty}(\Omega)}$. By the Neumann condition $\partial_{\nu} w=-(m \cdot \nu) F\left(w_{t}\right)$ on $\Gamma_{+}$and Lemma 5 , the following estimate is true (see $[27$, p. 471] for a proof):

$$
2(\Delta w, m \cdot \nabla w) \leq(n-2)\|\nabla w\|^{2}+R^{2} \int_{\Gamma_{+}}(m \cdot \nu) F^{2}\left(w_{t}\right) d \Gamma
$$

Copyright $@$ ( ) by SIAM. Unauthorized reproduction of this article is prohibited. 
Substituting (A.5) into (A.3) gives us

$$
\dot{\rho}(t) \leq-2 E(t)+\int_{\Gamma_{+}} m \cdot \nu\left[R^{2} F^{2}\left(w_{t}\right)-(n-1) F\left(w_{t}\right) w+\left|w_{t}\right|^{2}\right] d \Gamma .
$$

The mapping $H_{\Gamma_{0}}^{1}(\Omega) \rightarrow L^{2}\left(\Gamma_{+}\right)$being continuous, there is some constant $\beta>0$ such that

$$
\int_{\Gamma_{+}} m \cdot \nu|w|^{2} d \Gamma \leq \beta\|\nabla w\|^{2}
$$

By (A.7) and the Cauchy inequality we prove that

$$
\left|(n-1) \int_{\Gamma_{+}} m \cdot \nu F\left(w_{t}\right) w d \Gamma\right| \leq E(t)+\frac{(n-1)^{2} \beta}{2} \int_{\Gamma_{+}} m \cdot \nu F^{2}\left(w_{t}\right) d \Gamma .
$$

Substituting (A.8) into (A.6) and then (A.6) into (A.4) gives us the following:

$$
\begin{aligned}
& \dot{E}_{\epsilon}(t) \leq-\epsilon E^{\frac{p+1}{2}}(t) \\
& \quad+\int_{\Gamma_{+}} m \cdot \nu\left\{\epsilon E^{\frac{p-1}{2}}(t)\left[\left(R^{2}+\frac{(n-1)^{2} \beta}{2}\right) F^{2}\left(w_{t}\right)+\left|w_{t}\right|^{2}\right]-\frac{1}{2} F\left(w_{t}\right) w_{t}\right\} d \Gamma .
\end{aligned}
$$

We decompose $\Gamma_{+}$into two disjoint sets $\Gamma_{1}$ and $\Gamma_{2}$ which may vary with time:

$$
\Gamma_{1}=\left\{x \in \Gamma_{+}|| w_{t}(x, t) \mid<M_{1}\right\}, \quad \Gamma_{2}=\left\{x \in \Gamma_{+}|| w_{t}(x, t) \mid \geq M_{1}\right\} .
$$

For all $\epsilon$ satisfying

$$
\epsilon \leq \epsilon_{3}=\frac{C_{1}}{2 E^{\frac{p-1}{2}}(0)\left[1+\left(R^{2}+\beta(n-1)^{2} / 2\right) C_{2}^{2}\right]},
$$

we have

$$
\int_{\Gamma_{1}} m \cdot \nu\left\{\epsilon E^{\frac{p-1}{2}}(t)\left[\left(R^{2}+\frac{(n-1)^{2} \beta}{2}\right) F^{2}\left(w_{t}\right)+\left|w_{t}\right|^{2}\right]-\frac{1}{2} F\left(w_{t}\right) w_{t}\right\} d \Gamma \leq 0 .
$$

Notice that $C_{1}$ and $C_{2}$ are defined in (1.3).

It is easy to see that, from (1.3),

$$
\left|F\left(w_{t}\right)\right| \leq M_{1}^{-1} M_{2}\left|w_{t}\right| \forall x \in \Gamma_{2} .
$$

This implies that, $\forall \epsilon$ satisfying

$$
\int_{\Gamma_{2}} m \cdot \nu\left\{\epsilon E^{\frac{p-1}{2}}(t)\left[\left(R^{2}+\frac{(n-1)^{2} \beta}{2}\right) F^{2}\left(w_{t}\right)+\left|w_{t}\right|^{2}\right]-\frac{1}{2} F\left(w_{t}\right) w_{t}\right\} d \Gamma
$$

$$
\leq \epsilon E^{\frac{p-1}{2}}(t) \int_{\Gamma_{2}} m \cdot \nu\left|w_{t}\right|^{2} d \Gamma-\frac{1}{4} \int_{\Gamma_{2}} m \cdot \nu F\left(w_{t}\right) w_{t} d \Gamma
$$

Copyright (c) by SIAM. Unauthorized reproduction of this article is prohibited. 
Since $F$ is monotone increasing, by setting $F_{\min }=\min \left(F\left(M_{1}\right),\left|F\left(-M_{1}\right)\right|\right)$ we have

$$
\left|F\left(w_{t}\right)\right| \geq F_{\min } \forall x \in \Gamma_{2} .
$$

Let us set $s=\frac{2}{p+1} \forall p>1$. By the Hölder inequality we get

$$
\begin{aligned}
& \int_{\Gamma_{2}} m \cdot \nu\left|w_{t}\right|^{2} d \Gamma \leq F_{\min }^{-s} \int_{\Gamma_{2}} m \cdot \nu\left|w_{t}\right|^{2-s}\left(F\left(w_{t}\right) w_{t}\right)^{s} d \Gamma \\
& \leq F_{\min }^{-s}\left(\int_{\Gamma_{2}} m \cdot \nu\left|w_{t}\right|^{\frac{2-s}{1-s}} d \Gamma\right)^{1-s}\left(\int_{\Gamma_{2}} m \cdot \nu F\left(w_{t}\right) w_{t} d \Gamma\right)^{s} .
\end{aligned}
$$

By (A.17) and the Young inequality we get

$$
\begin{gathered}
E^{\frac{p-1}{2}}(t) \int_{\Gamma_{2}} m \cdot \nu\left|w_{t}\right|^{2} d \Gamma \leq \\
F_{\text {min }}^{-s} R^{1-s}\left[\delta(1-s) E^{\frac{p+1}{2}}(t) \int_{\Gamma_{2}}\left|w_{t}\right|^{\frac{2-s}{1-s}} d \Gamma+s(1 / \delta)^{\frac{1-s}{s}} \int_{\Gamma_{2}} m \cdot \nu F\left(w_{t}\right) w_{t} d \Gamma\right] \forall \delta>0 .
\end{gathered}
$$

As $\left(w^{0}, w^{1}\right) \in \mathcal{D}(W)$, we have $\left\|\nabla w_{t}\right\| \leq\left\|W\left(w^{0}, w^{1}\right)\right\|_{X} \forall t \geq 0$. Hence $w_{t} \in$ $H_{\Gamma_{0}}^{1}(\Omega)$. By the boundary trace imbedding theorem [1, Theorem 5.36, p. 164] we have continuous imbedding $H_{\Gamma_{0}}^{1}(\Omega) \rightarrow L^{\frac{2-s}{1-s}}\left(\Gamma_{+}\right)$, i.e., there exists some constant $\alpha_{2}$ such that

$$
\left(\int_{\Gamma_{2}}\left(\left|w_{t}\right|^{\frac{2-s}{1-s}} d \Gamma\right)^{\frac{1-s}{2-s}} \leq \alpha_{2}\left\|\nabla w_{t}\right\| \quad \forall p>1 \text { if } n=2 \text { and } \forall p \geq 2 \text { if } n=3\right. \text {, respectively. }
$$

For all $\epsilon$ satisfying

$$
\epsilon \leq \epsilon_{5}=\frac{F_{\min }}{4 s}\left(\frac{1}{3(1-s) R}\right)^{\frac{1-s}{s}}\left(\alpha_{2}\left\|W\left(w^{0}, w^{1}\right)\right\|_{X}\right)^{-\frac{2-s}{s}},
$$

there exists some $\delta>0$ such that

$$
\epsilon E^{\frac{p-1}{2}}(t) \int_{\Gamma_{2}} m \cdot \nu\left|w_{t}\right|^{2} d \Gamma \leq \frac{\epsilon}{3} E^{\frac{p+1}{2}}(t)+\frac{1}{4} \int_{\Gamma_{2}} m \cdot \nu F\left(w_{t}\right) w_{t} d \Gamma
$$

Taking $\epsilon=\min _{1 \leq i \leq 5} \epsilon_{i}$ and substituting (A.19) into (A.15) and then (A.15) and (A.12) into (A.9) allows us to prove the following:

$$
\dot{E}_{\epsilon}(t) \leq-\frac{2 \epsilon}{3} E^{\frac{p+1}{2}}(t) \forall t \geq 0 .
$$

The assertion (i) and (A.20) imply the assertion (ii):

$$
\dot{E}_{\epsilon}(t) \leq-\epsilon\left(\frac{2}{3}\right)^{\frac{p+3}{2}} E_{\epsilon}^{\frac{p+1}{2}}(t) \forall t \geq 0 .
$$

Hence the claim is proved and the proof of Theorem 3 is complete.

Acknowledgment. The authors would like to thank the reviewers and the editors for useful suggestions to improve the paper.

Copyright $\odot$ by SIAM. Unauthorized reproduction of this article is prohibited. 


\section{REFERENCES}

[1] R. Adams and J. Fournier, Sobolev Spaces, Academic Press, Singapore, 2009.

[2] V. BARBU, Nonlinear semigroups and differential equations in Banach space, Noordhoff International Publishing, Leyden, The Netherlands, 1976.

[3] H. BreZis, Operateurs Maximaux Monotones et Semigroupes de Contractions dans les Espaces de Hilbert, North-Holland, Amsterdam, 1973.

[4] G. Chen, Energy decay estimates and exact boundary value controllability for the wave equation in a bounded domain, J. Math. Pures Appl., 58 (1979), pp. 249-273.

[5] B. Chentouf, C. Z. Xu, And G. Sallet, On the stabilization of a vibrating equation, Nonlinear Anal., 39 (2000), pp. 537-558.

[6] F. Conrad And O. Morgul, On the stabilization of a flexible beam with a tip mass, SAIM J. Control Optim., 36 (1998), pp. 1962-1986.

[7] F. Conrad And M. Pierre, Stabilization of Euler-Bernoulli Beam by Nonlinear Boundary Feedback, Rapport de recherche INRIA, RR-1235 (1990), pp. 1-15, http://hal.inria.fr/ inria-00075324/PDF/RR-1235.pdf.

[8] J. M. Coron, B. D'AndréA-Novel, And G. Bastin, A strict Lyapunov function for boundary control of hyperbolic systems of conservation laws, IEEE Trans. Automat. Control, 52 (2007), pp. 2-11.

[9] M. G. Crandall And A. Pazy, Semigroups of nonlinear contractions and dissipative sets, J. Funct. Anal., 3 (1969), pp. 376-418.

[10] C. M. Dafermos And M. Slemrod, Asymptotic behavior of nonlinear contraction semigroups, J. Funct. Anal., 13 (1973), pp. 97-106.

[11] J. M. Greenberg And T. T. Li, The effect of boundary damping for the quasilinear wave equation, J. Differential Equations, 52 (1984), pp. 66-75.

[12] B. Z. Guo AND Z. H. Luo, Stability analysis of a hybrid system arising from feedback control of flexible robots, Jpn. J. Ind. Appl. Math., 13 (1996), pp. 417-434.

[13] A. Haraux, Nonlinear Evolution Equations - Global Behavior of Solutions, Lecture Notes in Mathematics, Springer-Verlag, New York, 1981.

[14] A. Haraux, Systèmes dynamiques dissipatifs et applications, Collection Recherche en Mathématiques Appliquées sous la direction de P.G. Ciarlet et J.-L. Lions, Masson, John Wiley \& Sons, Singapore, 1991.

[15] V. Komornik, Exact Controllability and Stabilization - The Multiplier Method, Research in Applied Mathematics, P. G. Ciarlet and J. L. Lions, eds., John Wiley \& Sons, Singapore, 1994.

[16] V. Komornik And E. Zuazua, A direct method for the boundary stability of the wave equation, J. Math. Pures Appl., 69 (1990), pp. 33-54

[17] I. LASIECKA, Stabilisation of wave and plate-like equations with nonlinear dissipation on the boundary, J. Differential Equations, 79 (1989), pp. 340-381.

[18] Z.H. Luo, B.Z. Guo, AND O. Morgul, Stability and Stabilization of Infinite-Dimensional Systems with Applications, Communications and Control Engineering, Springer-Verlag, New York, 1999.

[19] S. Micu And A. F. Pazoto, Almost periodic solutions for a weakly dissipative hybrid system, Math. Control Relat. Fields, 4 (2014), pp. 101-113.

[20] C. Prieur, S. Tarbouriech, and J. M. Gomes da Silva, Jr., Wave equation with conebounded control laws, IEEE Trans. Automat. Control, 61 (2016), pp. 3452-3463.

[21] B. RAO, Stabilisation du modèle SCOLE par un contrôle a pariori borné, Série I, C.R. Acad. Sci. Paris, 316 (1993), pp. 1061-1066.

[22] B. RAO, Stabilization of Kirchhoff plate equation in star-shaped domain by nonlinear boundary feedback, Nonlinear Anal. 20 (1993), pp. 605-626.

[23] S. Saperstone, Semidynamical systems in infinite-dimensional spaces, Appl. Math. Sci., 37, 1981.

[24] M. Slemrod, Feedback stabilization of a linear control system in Hilbert space with an a priori bounded control, Math. Control Signals Systems, 2 (1989), pp. 265-285.

[25] M. Tucsnak And G. Weiss, Observation and Control for Operator Semigroups, Birkhäser, Boston, 2009.

[26] J. Vancostenoble and P. Martinez, Optimality of energy estimates for the wave equation with nonlinear boundary velocity feedbacks, SIAM J. Control Optim., 39 (2000), pp. $776-797$.

[27] E. ZUAZUA, Uniform stabilization of the wave equation by nonlinear boundary feedback, SIAM J. Control Optim., 28 (1990), pp. 466-477.

Copyright (c) by SIAM. Unauthorized reproduction of this article is prohibited. 